\title{
Fast Neutron Capture Reaction Data Measurement of Minor Actinides for De- velopment of Nuclear Transmutation Systems
}

\author{
Tatsuya Katabuchi ${ }^{1, *}$, Osamu Iwamoto $^{2}$, Jun-ichi Hori $^{3}$, Atsushi Kimura ${ }^{2}$, Nobuyuki Iwamoto $^{2}$, Shoji Nakamura ${ }^{2}$, Yuji \\ Shibahara $^{3}$, Kazushi Terada $^{2}$, Gerard Rovira ${ }^{1}$, and Shota Matsuura ${ }^{1}$. \\ ${ }^{1}$ Tokyo Instutute of Technology, Laboratory for Advanced Nuclear Energy. \\ ${ }^{2}$ Japan Atomic Energy Agency. \\ ${ }^{3}$ Kyoto University.
}

\begin{abstract}
A research project entitled "study on accuracy improvement of fast-neutron capture reaction data of long-lived MAs for development of nuclear transmutation systems" is ongoing as a joint collaboration, including Tokyo Tech, Japan Atomic Energy Agency and Kyoto University in Japan. This project focuses on neutron capture reaction of minor actinides ${ }^{237} \mathrm{~Np},{ }^{241} \mathrm{Am}$ and ${ }^{243} \mathrm{Am}$, in the fast neutron energy region. The final goal of this project is to improve the neutron capture cross sections utilizing a high-intensity neutron beam from a spallation neutron source of the Japan Proton Research Complex. The overview of the project and the current progress are presented.
\end{abstract}

\section{Introduction}

Long-lived minor actinides (MA) in nuclear waste from nuclear power plants are a serious issue in the nuclear industries. Geological disposal of high-level nuclear waste has been considered as the most feasible option but choosing a disposal site was found to be a very difficult task in many countries. To solve or mitigate this issue, researchers have suggested nuclear transmutation, in which longlived radionuclides are transmuted into stable or shorterlived nuclides via neutron-induced nuclear reactions. Development of nuclear transmutation systems such as an accelerator-driven system (ADS) requires accurate neutron nuclear reaction data. In most of the proposed ADS, a subcritical system sustained by neutron input from an accelerator-based neutron source is adopted. The nuclear data required for the design of ADS include many neutroninduced reaction channels of various nuclides including MAs. Iwamoto et al. carried out a sensitivity and uncertainty analysis [1] with the latest version of the Japanese Evaluated Nuclear Data Library (JENDL-4.0)[2]. The results showed that the uncertainties of the capture cross section data of ${ }^{237} \mathrm{~Np},{ }^{241} \mathrm{Am}$ and ${ }^{243} \mathrm{Am}$ are sizable components to uncertainties of calculated reactor physics parameters such as criticality or void reactivity. Thus, to improve reactor physics calculation, more accurate neutron capture cross section data of the MAs are required.

This nuclear data demand motivated us to start a research project entitled "Study on accuracy improvement of fast-neutron capture reaction data of long-lived MAs for development of nuclear transmutation systems". The project started in 2017 as a joint collaboration between

*e-mail: buchi@lane.iir.titech.ac.jp
Tokyo Tech, Japan Atomic Energy Agency and Kyoto University. This project focuses on the neutron capture reaction of MAs, especially ${ }^{237} \mathrm{~Np},{ }^{241} \mathrm{Am}$ and ${ }^{243} \mathrm{Am}$, in the fast neutron energy region. The final goal of this project is to improve the accuracies of the neutron capture cross sections of ${ }^{237} \mathrm{~Np},{ }^{241} \mathrm{Am}$ and ${ }^{243} \mathrm{Am}$ employing a highintensity neutron beam from a spallation neutron source of the Japan Proton Accelerator Research Complex (JPARC) [3] that reduces uncertainties of measurement significantly. The Accurate Neutron Nucleus Reaction Measurement Instrument (ANNRI) has been built as a neutron beam line for nuclear data measurement in the Materials and Life Science Experimental Facility (MLF) of J-PARC [4, 5]. A lay-out of ANNRI is depicted in Fig. 1. Although many nuclear data measurements have been already conducted using ANNRI successfully [6-11], this project aims to improve ANNRI, focusing on neutron capture cross section measurement of MAs in the fast neutron energy region, particularly $0.5-500 \mathrm{keV}$. To achieve the goal, the project consists of four tasks: (1) development of a neutron beam filter system in J-PARC, (2) neutron capture cross section measurement using the ANNRI NaI(Tl) detectors, (3) sample characteristic assay, and (4)theoretical reaction model study. Detail of each task is described in the following section.

\section{Project tasks}

\subsection{Neutron beam filter}

The neutron beam filter is designed to solve the so-called double-pulse beam issue in J-PARC. Neutrons are produced in the J-PARC/MLF spallation target at a repetition 


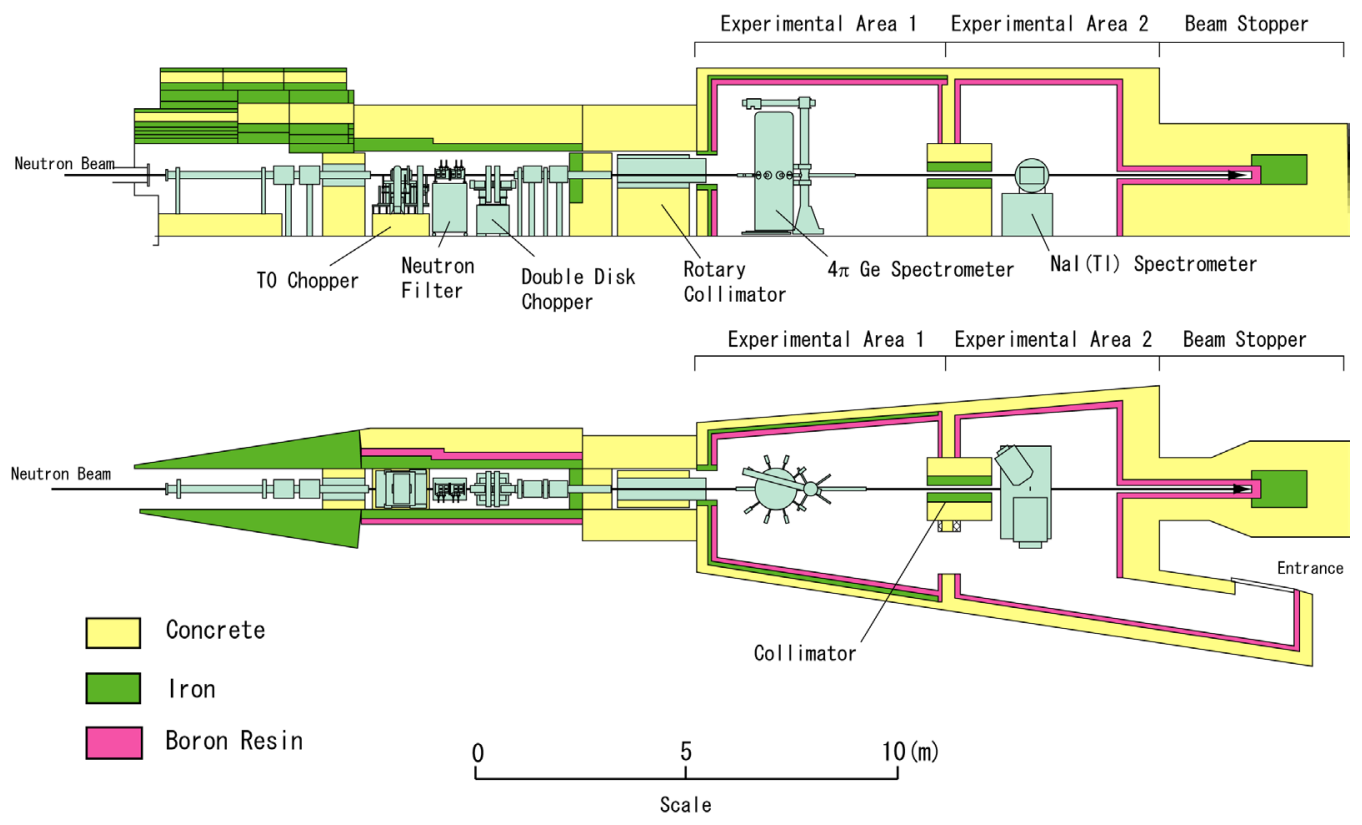

Figure 1. Accurate Neutron Nucleus Reaction Measurement Instrument (ANNRI)

rate of $25 \mathrm{~Hz}$. The proton accelerator is operated at a special operational pattern that two 100 -ns wide proton beam pulses separated by $600 \mathrm{~ns}$ are injected into the spallation target every $40 \mathrm{~ms}$. The reason why two proton pulses are injected is to simply increase the thermal neutron beam flux to satisfy users' demand. Among more than 20 neutron beam lines of MLF, most of the users use only low energy neutrons (thermal or cold). The 600-ns double pulse beam structure disappear in the neutron slow-down process in moderators and thereby does not affect a neutron time-of-flight (TOF) spectrum in the thermal energy region. However, in the higher energy region $(>10 \mathrm{eV})$, the double pulse beam is crucially problematic [12]. Resonance peaks in a TOF spectrum become doublet due to the double pulse beam structure. This makes resonance analysis more complicated, requiring the implementation of a resolution function of the double-pulse beam into resonance analysis.

Situation is even worse in the continuum region above the resolved resonance region. The cross section is a smooth function of neutron energy. Neutrons that have two different energies associated with two different neutron burst times caused by the double proton beam pulses reach a sample at the same time. It is difficult to deconvolute the measured neutron capture yield into single-pulse components.

In order to solve the double-pulse beam issue in $\mathrm{J}$ PARC/MLF, a neutron beam filter system is under development in ANNRI. The neutron beam filter technique has been often used in nuclear reactors, to make mono or quasi-mono energetic neutron beams. A total cross section minimum at an s-wave resonance is used as an energy window of neutron energy filtering. Combining optimal filter materials, a neutron energy spectrum can be tailored to be mono-energetic. We plan to employ the neu- tron beam filter technique to separate two different neutron energy components at the same TOF channels. The sample position of ANNRI is $27.9 \mathrm{~m}$ from the neutron source. The neutron beam filter system is placed in a rotary collimator at a flight length of around $18 \mathrm{~m}$. The selected filter materials are $\mathrm{Fe}, \mathrm{Bi}, \mathrm{Al}, \mathrm{Si}, \mathrm{Cr}$ and $\mathrm{Sc}_{2} \mathrm{O}_{3}$. They have resonances which are suitable for filtering neutrons in keV energy region. The neutron filter materials have been fabricated and tested in neutron beam experiments at Tokyo Tech and J-PARC. In the Tokyo Tech experiments, a neutron beam from the ${ }^{7} \mathrm{Li}(\mathrm{p}, \mathrm{n})^{7} \mathrm{Be}$ reaction induced by a proton beam from a Pelletron accelerator bombarding a $\mathrm{Li}$ target was used. Transmission spectra were measured by the TOF method with a ${ }^{6} \mathrm{Li}$ glass scintillator. Examples of measured neutron spectrum for $5-\mathrm{cm}$ and $10-\mathrm{cm}$ thick Fe filters are shown in Fig. 2. The peak is observed at $24 \mathrm{keV}$, the neutron resonance energy of ${ }^{56} \mathrm{Fe}$. In the J-PARC experiments, transmission neutron spectra were measured by detecting the ${ }^{10} \mathrm{~B}(\mathrm{n}, \alpha \gamma)^{7} \mathrm{Li}$ prompt $\gamma$-rays from a boron sample with the $\mathrm{NaI}(\mathrm{Tl})$ detector. Figure 3 shows a neutron TOF spectrum for $10-\mathrm{cm}$ thick Fe filter. The neutron spectrum is filtered well to form a $24 \mathrm{keV}$ peak.

\section{2 $\mathrm{Nal}(\mathrm{TI})$ detectors for neutron capture measurement}

Although ANNRI has two different $\gamma$-ray detection systems: Ge detector array and $\mathrm{NaI}(\mathrm{Tl})$ detectors, this project mainly uses the $\mathrm{NaI}(\mathrm{Tl})$ detectors. A $\mathrm{NaI}(\mathrm{Tl})$ detector has a much faster response property than a Ge detector. Thus, the $\mathrm{NaI}(\mathrm{Tl})$ detectors are suited for TOF measurement in the fast neutron energy region. On the other hand, the Ge detector array cannot be used above $1 \mathrm{keV}$ due to slow detector response. For capture cross section measurement in 


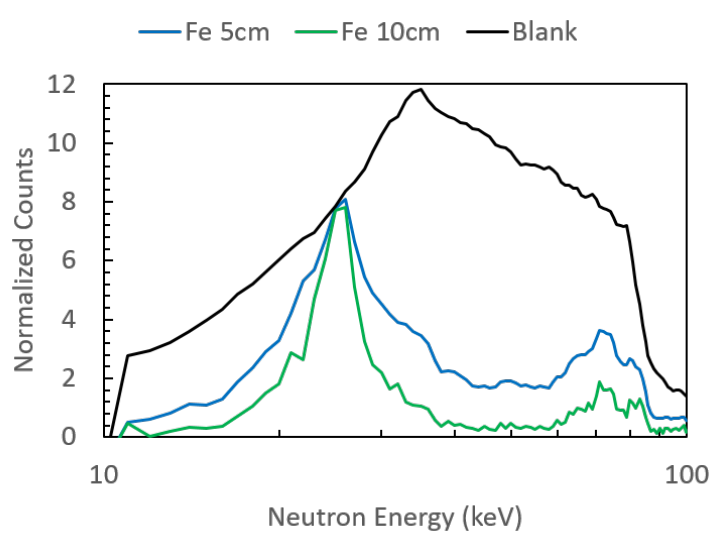

Figure 2. Transmission neutron spectra after 5- and 10-cm thick Fe measured in Tokyo Tech.

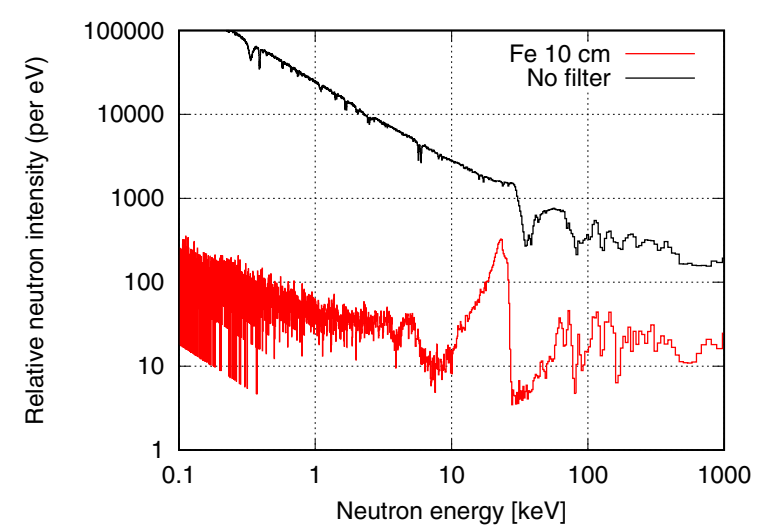

Figure 3. Transmission neutron spectra after 5- and 10-cm thick Fe measured in J-PARC.

the fast energy region, the ANNRI-NaI(Tl) detectors are chosen.

Figure 4 shows the ANNRI-NaI(Tl) detectors. The ANNRI-NaI(Tl) detectors consist of two differently-sized $\mathrm{NaI}(\mathrm{Tl})$ detectors. The larger detector (crystal size: 330mm diam. $\times 203-\mathrm{mm}$ long) is placed at a detection angle of $90^{\circ}$ as respect from the beam axis. The smaller detector (203-mm diam. $\times 203-\mathrm{mm}$ long) is at an angle of $125^{\circ}$. The detectors are shielded from background $\gamma$ rays and neutrons with various shielding materials such as lead, borated polyethylene and paraffin, cadmium and ${ }^{6} \mathrm{Li}-$ enriched lithium compounds. A sample is inserted into a carbon fiber beam duct and set at a flight length of $27.9 \mathrm{~m}$. The beam duct is filled with helium gas to reduce neutron scattering background by air.

Fast signal processing is required for data acquisition in the fast neutron energy region. Data acquisition with traditional peak-hold analog-to-digital converters is too slow to process high-counting-rate signals caused by the intense neutron beam of J-PARC. The current data acquisition system is based on a pulse-width analysis method recently developed by our group [13]. The pulse-width analysis method successfully works in fast energy region

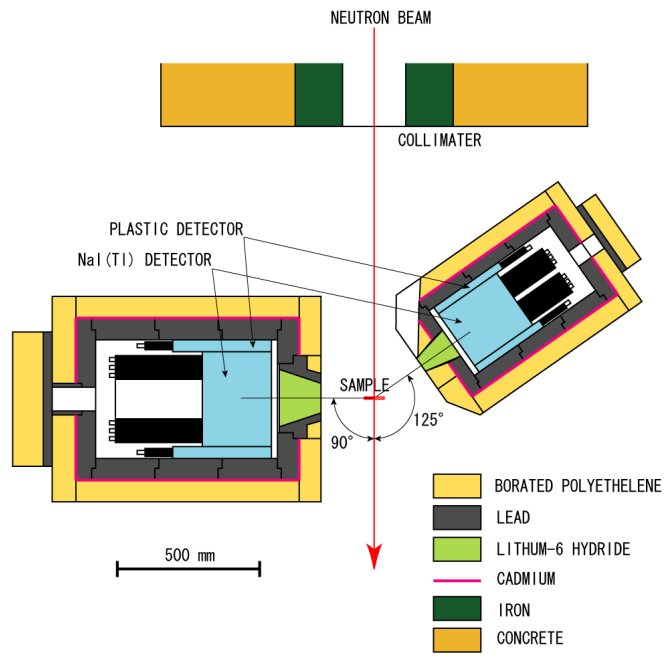

Figure 4. ANNRI-NaI(Tl) detectors.

but a disadvantage is poor energy resolution. The present project focuses on not only capture cross sections but also capture prompt $\gamma$-ray spectra. Capture prompt $\gamma$-ray spectra give more information on reaction mechanism that is very useful for theoretical study. To improve the energy resolution, the data acquisition system is being replaced with a waveform digitizer (CAEN V1720) that enables to store the waveform of the signal of each event and allow for analyzing signal data off-line.

In addition, a sample rotational system that can tilt a sample with respect to the beam axis has been installed in ANNRI. Tilting a sample increases the effective sample thickness which neutrons pass through. Accordingly, measurement with different sample thicknesses can be carried out without purchasing multiple samples.

\subsection{Sample characteristics assay}

Uncertainties originating from samples are main sources of systematic uncertainties of cross sections. The sample characteristic assay, particularly, precise isotope mass spectrometry lowers sample systematic uncertainties. Isotope compositions are sometimes off from the rated values. Radioactive samples often have impurities not listed in a certificate sheet.

In this project, a thermal ionization mass spectrometer (TIMS) of the the Institute for Integrated Radiation and Nuclear Science of Kyoto University is used to analyze the isotope compositions and chemical impurities of samples. All MA samples for measurement at J-PARC are sealed in containers. For analysis, unsealed MA solution taken from the same batch as the sealed MA samples were already prepared. The Kyoto-University TIMS was upgraded to improve its vacuum system and stability control of the beam intensity in the past two years. Actual spectroscopy of the MA samples is planned to start in 2019. The target precision is less than $1 \%$. 


\subsection{Theoretical reaction model study}

Details of the theoretical reaction model study in this project are described in another contribution by Iwamoto et al [14]. Only brief description is given here. This task aims improving evaluation method of MA nuclear data, based on both capture cross sections and prompt $\gamma$ ray spectra measured with the ANNRI-NaI(Tl) detectors. The theoretical nuclear reaction model CCONE $[15,16]$ is employed to evaluate the capture cross sections of MA nuclides. In addition, capture $\gamma$-ray spectra are included in analysis to determine the model parameters of $\gamma$-ray strength function. To compare theoretical model calculations with experimental data of $\gamma$-ray spectra, a method to calculate pulse-height spectra of the $\mathrm{NaI}(\mathrm{Tl})$ detectors is under development. Detector response functions of the $\mathrm{NaI}(\mathrm{Tl})$ detectors were calculated by the Monte Carlo simulation code PHITS [17]. After that, a prompt $\gamma$-ray spectrum calculate with CCONE is folded with the detector response functions to give the pulse-height spectrum for comparison. Test experiments using a standard Au foil at ANNRI resulted in good agreement.

\section{Summary}

A project "study on accuracy improvement of fast-neutron capture reaction data of long-lived MAs for development of nuclear transmutation systems" is ongoing as a joint research collaboration of the three institutes, Tokyo Tech, JAEA and Kyoto University. The project focuses on neutron capture reaction of Mas, especially ${ }^{237} \mathrm{~Np},{ }^{241} \mathrm{Am}$ and ${ }^{243} \mathrm{Am}$, in the fast neutron energy region. An intense neutron beam from a spallation source of J-PARC will be employed to improve the neutron capture data of MA. The project stated in 2017. The past two years were spent for development and preparation. Actual measurement using ANNRI-NaI(Tl) detectors at J-PARC will start in 2019.

\section{Acknowledgments}

This work is supported by the Innovative Nuclear Research and Development Program from the Ministry of
Education, Culture, Sports, Science and Technology of Japan.

\section{References}

[1] H. Iwamoto et al., Journal of Nuclear Science Technology 50 (2013) 856.

[2] K. Shibata et al., Journal of Nuclear Science Technology 48 (2011) 1.

[3] F. Maekawa et al., Nuclear Instruments and Methods in Physics Research A 620 (2010) 159.

[4] M. Igashira et al., Nuclear Instruments and Methods in Physics Research A 600 (2009) 332.

[5] Y. Kiyanagi et al., Journal of Korean Physical Society 59 (2011) 1781.

[6] A. Kimura et al., Journal of Nuclear Science Technology 49 (2012) 708.

[7] K. Hirose et al., Journal of Nuclear Science Technology $\mathbf{5 0}$ (2013) 188.

[8] K. Terada et al., Nuclear Data Sheets 119 (2014) 147.

[9] T. Katabuchi et al., Physical Review C 91 (2015) 037603.

[10] K. Terada et al., Journal of Nuclear Science Technology 55 (2018) 1198.

[11] A. Kimura et al., Journal of Nuclear Science Technology 56 (2019) 479.

[12] K. Kino et al., Nuclear Instruments and Methods in Physics Research A 626-627 (2011) 58-66.

[13] T. Katabuchi et al., Nuclear Instruments and Methods in Physics Research A 764 (2014) 369-377.

[14] N. Iwamoto et al., Evaluation of Gamma-ray Strength Function Based on Measured Gamma-ray Pulse-height Spectra in Time-of-flight Neutron Capture Experiments, Proceedings of ND2019, S284.

[15] O. Iwamoto, Journal of Nuclear Science Technology 44 (2007) 687.

[16] O. Iwamoto et al., Nuclear Data Sheets 131 (2016) 259.

[17] T. Sato et al., Journal of Nuclear Science Technology 55 (2018) 684. 